\title{
AN APPLICATION OF THE NONSELFADJOINT OPERATORS THEORY IN THE STUDY OF STOCHASTIC PROCESSES
}

\author{
LYAZID ABBAOUI AND LATIFA DEBBI
}

Received 11 May 2003 and in revised form 1 March 2004

The theory of operator colligations in Hilbert spaces gives rise to certain models for nonselfadjoint operators, called triangular models. These models generalize the spectral decomposition of selfadjoint operators. In this paper, we use the triangular model to obtain the correlation function (CF) of a nonstationary linearly representable stochastic process for which the corresponding operator is simple, dissipative, nonselfadjoint of rank 1 , and has real spectrum. As a generalization, we represent the infinitesimal correlation function (ICF) of a nonhomogeneous linearly representable stochastic field in which at least one of the operators has real spectrum.

\section{Introduction}

Kolmogorov, Karhunen, and others have considered stochastic fields of second order as hypersurfaces in a Hilbert space. This consideration makes it possible to use certain functional tools. The application of spectral theory of the selfadjoint operator in the study of stochastic homogeneous fields gives immediately the spectral decomposition of the field and of its correlation function (CF). Yantsevich and Livšic introduced a class of centered Hilbertian nonhomogeneous stochastic fields tied to bounded nonselfadjoint operators, known as linearly representable fields. In $[1,8]$, we find necessary and sufficient conditions for stochastic fields to be linearly representable. In their monograph [8], Livšic and Yantsevich have used the infinitesimal correlation function (ICF) to represent the CF of a linearly representable process with simple dissipative operator of rank 1. Later, Abbaoui, Kirchev, and Zolotarev have obtained analogous results for a broader class of nonhomogeneous fields $[1,7,9]$.

We consider a stochastic process which is a solution of the system

$$
i \frac{d Z}{d t}+A Z=0, \quad Z(0)=Z_{0}
$$

where $A$ is a simple dissipative operator of rank 1 . The simplicity is not a restrictive condition because every operator could be written as an orthogonal sum of a simple operator and a selfadjoint one [5]. 
The CF was given as a sum of a kernel and an indefinite integral of the ICF.

Livšic and Yantsevich studied two cases:

(1) the operator $A$ is complete;

(2) the spectrum of the operator $A$ is concentrated at zero.

The utilization of the triangular model $[2,3,8]$ takes the study from the Hilbert space $H_{Z} \subset L_{2}(\Omega)$ to the Hilbert space $L_{2}(0, l)$. In the two cases studied, the system is asymptotically damped, contrary to the case when the spectrum of the operator $A$ is real, not concentrated in a unique point.

In this paper, we investigate a third case when the spectrum is purely real nonconcentrated at zero. We begin by the case when the spectrum is concentrated at a unique point different from zero. Without difficulty, we find the CF when the spectrum is finite. But in the general case, we are confronted with the difficulty of integrating a function over a closed rectifiable curve enclosing nonisolated critical points. We give the CF and the ICF as limits of sequences of CFs and, respectively, ICFs, corresponding to a sequence of complex Gaussian stochastic processes.

In [1], Abbaoui generalized the study of Livšic and Yantsevich to the nonhomogeneous centered Hilbertian stochastic fields, admitting linear representation when the operators are complete or have zero as spectrum. Using the idea of stochastic process, we represent the ICF of the same stochastic field when at least one of the operators has purely real spectrum [4].

We note that the result obtained here is valid for stochastic fields as well as for curves or surfaces in arbitrary Hilbert spaces.

\section{Definitions and preliminaries}

Let $(\Omega, \digamma, P)$ be a probability space and let $Z(t), t \in \mathbb{R}$, be a centered Hilbertian stochastic field; that is, $M Z(t)=0$ and $M|Z(t)|^{2}<\infty$, where $M$ is the mathematical expectation. $H_{Z}$ is a subspace of $L_{2}(\Omega)$ spanned by the values of the stochastic variables $Z(t), t \in \mathbb{R}$.

Definition 2.1. The stochastic process $Z(t), t \in \mathbb{R}$, is said to be linearly representable if in $H_{Z}$ it can be written in the form

$$
Z(t)=\exp (i t A) Z_{0}
$$

where $Z_{0}$ is a fixed element of $H_{Z}$ and $A$ is a bounded linear operator defined in $H_{Z}$.

If $Z(t), t \in \mathbb{R}$, is linearly representable, then $H_{Z}$ is separable.

Definition 2.2. A function of a process $Z(t), t \in \mathbb{R}$, is called ICF if it exists as follows:

$$
W(t, s)=-\left.\frac{\partial V(t+\tau, s+\tau)}{\partial \tau}\right|_{\tau=0},
$$

where $V(t, s)=M Z(t) \overline{Z(s)}$ is the CF of $Z(t)$.

Let the operator $A$ in (2.1) be simple, dissipative, and nonselfadjoint of rank 1 with real spectrum. 
Lemma 2.3. The $C F V(t+\tau, s+\tau)$ of $Z(t), t \in \mathbb{R}$, admits a limit when $\tau$ tends to infinity. This limit, denoted by $V_{\infty}(t-s)$, is a positive semidefinite selfadjoint kernel depending on $(t-s)[8]$.

Using this lemma, we can represent the CF of this process by

$$
V(t, s)=V_{\infty}(t-s)+\int_{0}^{+\infty} W(t+\tau, s+\tau) d \tau,
$$

where

$$
W(t, s)=\langle 2 \operatorname{ImAZ}(t), Z(s)\rangle_{H_{Z}},
$$

$2 \operatorname{Im} A=(1 / i)\left(A-A^{*}\right)$ and $A^{*}$ is the adjoint of $A$.

We include $A$ in the colligation $\Theta=\left(A, H_{Z}, g, 1\right), g \in H_{Z}$ is the canal vector, hence

$$
\begin{gathered}
2 \operatorname{ImAZ}(t)=\langle Z(t), g\rangle_{H_{Z}} g, \\
W(t, s)=\Phi(t) \overline{\Phi(s)}, \\
\Phi(t)=\left\langle\exp (i t A) Z_{0}, g\right\rangle=-\frac{1}{2 \pi i} \oint_{\gamma} \exp (i t \lambda)\left\langle Z_{0},\left(A^{*}-\bar{\lambda} I\right)^{-1} g\right\rangle_{H_{Z}} d \lambda,
\end{gathered}
$$

where $\gamma$ is a rectifiable curve enclosing the spectrum of $A$.

The triangular model of $A$ is the operator $\hat{A}$ defined in $L^{2}(0, l)$ by

$$
\hat{A}(f)(x)=\alpha(x) f(x)+i \int_{x}^{l} f(t) d t
$$

where $l \in \mathbb{R}_{+}^{*}$ and $\alpha(x)$ is a nondecreasing bounded function of $x, 0 \leq x \leq l$.

It is proved in [3] that the image of the function $\alpha$ is the spectrum of $A$.

Replacing $A$ by its triangular model, we find

$$
\Phi(t)=-\frac{1}{2 \pi i} \oint_{\gamma} \exp (i t \lambda)\left\langle f_{0},\left(\hat{A}^{*}-\bar{\lambda} I\right)^{-1} \hat{g}\right\rangle_{L^{2}(0, l)} d \lambda,
$$

where $\hat{g}(x)=1$, for all $x \in(0, l)$, is the canal vector in the colligation $\widehat{\Theta}=\left(\hat{A}, L^{2}(0, l), \hat{g}, 1\right)$.

To calculate the function $f_{\lambda}=\left(\hat{A}^{*}-\bar{\lambda} I\right)^{-1} \hat{g}$, we consider the function $Y_{\lambda}(x)=$ $(\alpha(x)-\bar{\lambda}) f_{\lambda}(x)$. Such a function is a solution of the Cauchy problem

$$
\frac{d Y_{\lambda}}{d x}-i \frac{Y_{\lambda}}{(\alpha(x)-\bar{\lambda})}=0, \quad Y_{\lambda}(0)=1
$$

hence

$$
\begin{gathered}
f_{\lambda}(x)=(\alpha(x)-\bar{\lambda})^{-1} \exp \left(i \int_{0}^{x}(\alpha(x)-\bar{\lambda})^{-1} d \xi\right), \\
\Phi(t)=\int_{0}^{l} f_{0}(\xi) \Lambda_{\alpha}(t, \xi) d \xi \\
\Lambda_{\alpha}(t, \xi)=-\frac{1}{2 \pi i} \oint_{\gamma}(\alpha(x)-\bar{\lambda})^{-1} \exp \left(i t \lambda+i \int_{0}^{\xi}(\alpha(x)-\bar{\lambda})^{-1} d \zeta\right) d \lambda .
\end{gathered}
$$




\section{An application of the nonselfadjoint operators theory}

The problem now is to represent the function $\Lambda_{\alpha}(t, \xi)$. It is clear that the critical points of the integrating function are the values taken by $\alpha(\cdot)$ over $(0, \xi)$.

If the spectrum of $A$ contains only the point zero, then

$$
\Phi(t)=\int_{0}^{l} f_{0}(\xi) J_{0}(2 \sqrt{t \xi}) d \xi
$$

where $J_{0}(\cdot)$ is the Bessel function of the first kind of zero order. It is proved that in this case the process $Z(t)$ is asymptotically damped, that is, $V_{\infty}(t-s)=0$, so

$$
V_{0}(t, s)=\int_{0}^{+\infty} \Phi(t+\tau) \overline{\Phi(t+\tau)} d \tau .
$$

Theorem 2.4. Let $Z(t)$ be a Gaussian complex process and $M Z(t)=0$; then the $C F$ is a positive semidefinite selfadjoint kernel. Conversely, a given positive semidefinite selfadjoint kernel is the CF of some Gaussian complex process [6].

\section{Correlation function of a process with real spectrum not equal to zero}

3.1. $\sigma(A)$ contains a unique point different from zero. Let $\sigma(A)=\{c\}, c \neq 0$; then $\hat{A}=$ $c I+B$, where $I$ is the identity in $L^{2}(0, l)$ and $B$ is defined in the same space by

$$
(B f)(x)=i \int_{x}^{l} f(\xi) d \xi
$$

We have

$$
\exp (i t \hat{A})=\exp (\text { itc }) \exp (i t B)
$$

so

$$
V(t, s)=\exp (i c(t-s)) V_{0}(t, s)
$$

where $V_{0}(t, s)$ is given by $(2.11),(2.10)$.

3.2. $\sigma(A)$ finite. Let $\sigma(A)=\left\{c_{1}, c_{2}, \ldots, c_{m} / c_{k} \in \mathbb{R}, k=1,2, \ldots, m\right\}, c_{1}<c_{2}<\cdots<c_{m}$.

In this case, $\alpha(\cdot)$ is a simple function and is given by

$$
\alpha(\xi)=\sum_{j=1}^{m} c_{j} \chi_{j}(\xi)
$$

where $\chi_{j}=\chi_{\left[\xi_{j-1}, \xi_{j}\right.}$ is the characteristic function of the interval $\left[\xi_{j-1}, \xi_{j}[\right.$.

Applying the residue theorem, we find that

$$
\forall \xi \in\left[\xi_{k-1}, \xi_{k}\left[, \quad \Lambda_{\alpha}(t, \xi)=\sum_{\beta=1}^{k} \Lambda_{\alpha}^{(\beta)}(t, \xi),\right.\right.
$$


where

$$
\begin{aligned}
& \Lambda_{\alpha}^{(k)}(t, \xi)=\left[\exp \left(i t c_{k}+i \sum_{j=1}^{k-1}\left(c_{k}-c_{j}\right)^{-1} l_{j}\right)\right] \\
& \times\left[J_{0}\left(2 \sqrt{t l_{k}}\right)+\sum_{n=1}^{+\infty}\left(\left(-l_{k} t^{-1}\right)^{n / 2} J_{n}\left(2 \sqrt{t l_{k}}\right)\right)\right. \\
& \left.\times \sum_{k_{1}+\cdots+n k_{n}=n} \frac{1}{k_{1} ! \cdots k_{n} !} \prod_{\gamma=1}^{n}\left(i \sum_{j=1}^{k-1}\left(c_{k}-c_{j}\right)^{-\gamma-1} l_{j}\right)^{k_{\gamma}}\right], \\
& \Lambda_{\alpha}^{(\beta)}(t, \xi)=\left[\exp \left(i t c_{\beta}+i \sum_{j=1, j \neq \beta}^{k}\left(c_{\beta}-c_{j}\right)^{-1} l_{j}\right)\right] \\
& \times\left[\sum_{n=1}^{+\infty}-\left(c_{\beta}-c_{k}\right)^{-n}\left(-\frac{l_{\beta}}{t}\right)^{n / 2} J_{n}\left(2 \sqrt{t l_{\beta}}\right)\right. \\
& -\sum_{n=1}^{+\infty}\left(c_{\beta}-c_{k}\right)^{-n-1}\left(-l_{\beta} t^{-1}\right)^{(n+1) / 2} J_{n+1}\left(2 \sqrt{t l_{\beta}}\right) \\
& \left.\times \sum_{\nu=1}^{n} \sum_{k_{1}+\cdots+\nu k_{\nu}=\nu}\left(\frac{\left(c_{\beta}-c_{k}\right)^{\nu}}{k_{1} ! \cdots k_{\nu} !}\right) \prod_{\gamma=1}^{\nu}\left(i \sum_{j=1, j \neq \beta}^{k} l_{j}\left(c_{\beta}-c_{j}\right)^{-\gamma-1}\right)^{k_{\gamma}}\right] \quad \text { for } \beta<k \text {, }
\end{aligned}
$$

$l_{j}= \begin{cases}\xi-\xi_{k-1}, & \text { for } j=k, \\ \xi_{j}-\xi_{j-1}, & \text { otherwise. }\end{cases}$

The functions $j_{n}(x)=\sum_{k=0}^{+\infty}\left((-1)^{k}(x)^{2 k+n} / k !(k+n) !\right)$ and $j_{-n}(x)=(-1)^{n} j_{n}(x), n \geq 0$, are the Bessel functions of the first kind of orders $n$ and $-n$, respectively.

\section{3. $\sigma(A)$ arbitrary real.}

Theorem 3.1. Let $Z(t)=\exp (i t A) Z_{0}, Z_{0} \in H_{Z}$, be a Hilbertian linearly representable process such that $M Z(t)=0$ and $A$ is a simple, dissipative, and nonselfadjoint operator of rank 1 with real spectrum not concentrated in zero. Then there exists a sequence of linearly representable Gaussian complex processes $Z_{n}(t)=\exp \left(i t A_{n}\right) Z_{n 0}$, where $Z_{n 0} \in H_{Z_{n}}$ and $A_{n}$ is a simple, dissipative, and nonselfadjoint operator of rank 1 , defined in $H_{Z_{n}}$ with finite real spectrum such that

(1) the CF of $Z(t)$ is a simple limit of the sequence of CFs corresponding to the sequence of the processes $Z_{n}(t)$;

(2) the ICF of $Z(t)$ is a simple limit of the sequence of ICFs corresponding to the sequence of the processes $Z_{n}(t)$; 
154 An application of the nonselfadjoint operators theory

(3) the CF and the ICF of $Z_{n}(t)$ are given by

$$
\begin{gathered}
V_{n}(t, s)=V_{\infty}^{n}(t-s)+\int_{0}^{+\infty} \Phi_{n}(t+\tau) \overline{\Phi_{n}(s+\tau)} d \tau \\
W_{n}(t, s)=\Phi_{n}(t) \overline{\Phi_{n}(s)},
\end{gathered}
$$

where the functions $\Phi_{n}$ are given by

$$
\Phi_{n}(t)=\int_{0}^{l} f_{0}(\xi) \Lambda_{\alpha_{n}}(t, \xi) d \xi
$$

and $V_{\infty}^{n}(t-s)$ is a positive semidefinite selfadjoint kernel, where $\Lambda_{\alpha_{n}}(t, \xi)$ is the function given by (3.4), (3.5), (3.6), (3.7), and (3.8), with $c_{j}^{(n)}=\left(j / 2^{n}\right) \alpha_{\text {sup }}+\left(1-j / 2^{n}\right) \alpha_{\text {inf }}$ and $\alpha_{\text {sup }}=\sup \{\alpha(\xi), \xi \in(0, l)\}, \alpha_{\text {inf }}=\inf \{\alpha(\xi), \xi \in(0, l)\}, \xi_{j}^{(n)}, j=0,1, \ldots, 2^{n}$, are points of $(0, l)$ defined by

$$
\begin{gathered}
\xi_{j-1}^{(n)}=\inf \left\{\xi \in(0, l) \backslash j 2^{-n} \alpha_{\text {sup }}+\left(1-\frac{j}{2^{n}}\right) \alpha_{\text {inf }} \leq \alpha(\xi) \leq \frac{(j+1)}{2^{n}} \alpha_{\text {sup }}+\left(1-\frac{(j+1)}{2^{n}}\right) \alpha_{\text {inf }}\right\}, \\
\sigma\left(A_{n}\right) \subseteq\left\{c_{j}^{(n)}, j=1,2, \ldots, m_{n}\right\} .
\end{gathered}
$$

Proof. We construct a sequence of operators $\hat{A}_{n}, n \geq 1$, defined in $L^{2}(0, l)$ by

$$
\left(\hat{A}_{n} f\right)(x)=\alpha_{n}(x) f(x)+i \int_{x}^{l} f(\xi) d \xi,
$$

where $\alpha_{n}(\cdot)$ is a sequence of bounded simple functions uniformly converging to $\alpha(\cdot)$.

The operator $\hat{A}_{n}$ is bounded, dissipative, and nonselfadjoint of rank 1 and has finite spectrum spanned by the values taken by $\alpha_{n}(\cdot)$.

We will prove that the sequences of operators $\left\{\hat{A}_{n}\right\},\left\{\exp \left(i t \hat{A}_{n}\right)\right\}$ converge uniformly to $\hat{A}, \exp (i t \hat{A})$, respectively. In fact,

$$
\begin{aligned}
\left\|\hat{A} f-\hat{A}_{n} f\right\|^{2} & =\int_{0}^{l}\left|\alpha(x)-\alpha_{n}(x)\right|^{2}|f(x)|^{2} d x \\
& \leq \operatorname{Sup}_{x \in(0, l)}\left|\alpha(x)-\alpha_{n}(x)\right|^{2}\|f(x)\|^{2},
\end{aligned}
$$

so $\left\|\hat{A}-\hat{A}_{n}\right\| \leq \operatorname{Sup}_{x \in(0, l)}\left|\alpha(x)-\alpha_{n}(x)\right|$.

Let $\hat{B}_{n}=\hat{A}_{n}-\hat{A}$; then the sequence $\left\{\hat{B}_{n}\right\}$ converges uniformly to the null operator and we have

$$
\begin{aligned}
\left\|\exp \left(i t \hat{A}_{n}\right)-\exp (i t \hat{A})\right\| & =\left\|\exp \left(i t\left(\hat{A}+\hat{B}_{n}\right)\right)-\exp (i t \hat{A})\right\| \\
& \leq \sum_{k=0}^{+\infty} \frac{|t|^{k}}{k !}\left(\left(\left\|\hat{B}_{n}\right\|+\|\hat{A}\|\right)^{k}-\|\hat{A}\|^{k}\right) \\
& \leq \exp (|t|\|\hat{A}\|)\left(\exp \left(|t|\left\|\hat{B}_{n}\right\|\right)-1\right) .
\end{aligned}
$$


Let $h(t)=\exp (i t \hat{A}) f_{0}$, where $\hat{A}$ is the triangular model of $A$ and $f_{0}$ is the image of $Z_{0}$ by the isometric operator of the unitary equivalence between the colligation and the principal component of $\widehat{\Theta}$, and let $h_{n}(t)=\exp \left(i t \hat{A}_{n}\right) f_{0}$.

From the convergence of $\left\{\hat{A}_{n}\right\}$ and $\left\{\exp \left(i t \hat{A}_{n}\right)\right\}$, the sequences $\left\{h_{n}(t)\right\}$ and $\left\{\Phi_{n}(t)=\right.$ $\left.\left\langle h_{n}(t), \hat{g}\right\rangle\right\}$ converge, respectively, to $h(t)$ and $\Phi(t)$. We know that

$$
\begin{aligned}
\left|V_{n}(t, s)-V(t, s)\right| & =\left|\left\langle h_{n}(t), h_{n}(s)\right\rangle-\langle h(t), h(s)\rangle\right| \\
& \leq\left|\left\langle h_{n}(t), h_{n}(s)-h(s)\right\rangle-\left\langle h_{n}(t)-h(t), h(s)\right\rangle\right| \\
& \leq|| h_{n}(t)|||| h_{n}(s)-h(s)||+|| h(s)|||| h_{n}(t)-h(t)||, \\
\left|W_{n}(t, s)-W(t, s)\right| & =\left|\Phi_{n}(t) \overline{\Phi_{n}(s)}-\Phi(t) \overline{\Phi(s)}\right| \\
& \leq\left|\Phi_{n}(t)\right|\left|\Phi_{n}(s)-\Phi(s)\right|+|\Phi(s)|\left|\Phi_{n}(t)-\Phi(t)\right|,
\end{aligned}
$$

so $V_{n}(t, s) \rightarrow V(t, s)$ and $W_{n}(t, s) \rightarrow W(t, s)$, for all $t, s \in \mathbb{R}$.

The function $V_{n}(t, s)=\left\langle h_{n}(t), h_{n}(s)\right\rangle$ is a continuous positive semidefinite selfadjoint kernel and from Theorem 2.4 there exists a complex Gaussian process $Z_{n}(t)$ that has $V_{n}(t, s)$ as the CF. $V_{n}(t, s)$ and $W_{n}(t, s)$ are given by the formulas (3.5), (3.6), (3.7), (3.8), (3.9), (3.10), and (3.11).

The process $Z_{n}(t)$ is linearly representable. In fact, let $\hat{H}_{n}=\hat{H}_{h_{n}}$ (the subspace of $L^{2}(0, l)$ spanned by the vectors $\left.h_{n}(t), t \in \mathbb{R}\right)$; this subspace is invariant under $\hat{A}_{n}$; it is sufficient to prove that $\hat{A}_{n} h_{n}(t) \in \hat{H}_{n}$.

Let $U_{n}$ be the continuous extension of the operator $U_{n}^{0}$ defined in the linear span of all variables $Z_{n}(t), t \in \mathbb{R}$, by

$$
U_{n}^{0}\left(\sum_{k=1}^{m} c^{k} Z_{n}\left(t^{k}\right)\right)=\sum_{k=1}^{m} c^{k} h_{n}\left(t^{k}\right),
$$

so $f_{0}=U_{n} Z_{n 0}$ and $Z_{n}(t)=U_{n}^{-1} h_{n}(t)$.

Letting $A_{n}=U_{n}^{-1} \hat{A}_{n} U_{n}$, we have

$$
Z_{n}(t)=\exp \left(i t A_{n}\right) Z_{n 0}
$$

The restriction of $\hat{A}_{n}$ on $\hat{H}_{n}$ is a bounded, dissipative, and nonselfadjoint operator of rank 1 and has finite real spectrum contained in the image of the function $\alpha$. Since the operators $A_{n}$ and $\hat{A}_{n}$ are unitary-equivalent, then they have the same properties.

Remark 3.2. If the process $Z(t), t \in \mathbb{R}$, is asymptotically damped, that is, the kernel vanishes, then the CF is represented by

$$
V(t, s)=\int_{0}^{+\infty} \Phi(t+\tau) \overline{\Phi(s+\tau)} d \tau
$$

where $\Phi(t)$ is calculated previously. 
A generalization is given in [4] to represent an ICF of the linearly representable field:

$$
Z(x)=\exp \left(i \sum_{k=1}^{2} x_{k} A_{k}\right) Z_{0}
$$

where $x=\left(x_{1}, x_{2}\right) \in \mathbb{R}^{2}, Z_{0} \in H_{0}=\overline{2 \operatorname{Im} A_{1} 2 \operatorname{Im} A_{2} H_{Z}}$ with $\operatorname{dim} H_{0}=1$ and $A_{1}, A_{2}$ bounded, simple, dissipative, and nonselfadjoint operators such that $A_{1} A_{2}-A_{2} A_{1}=0$ and $A_{1}^{*} A_{2}-$ $A_{2} A_{1}^{*}=0$; the ICF is given by

$$
W(x, y)=\left.\frac{\partial V\left(x_{1}+\tau_{1}, x_{2}+\tau_{2}, y_{1}+\tau_{1}, y_{2}+\tau_{2}\right)}{\partial \tau_{1} \partial \tau_{2}}\right|_{\tau_{1=\tau_{2}=0}}
$$

We use the triangular model of a pair of operators, and by approximating these models, we represent the ICF in the case when at least one of the operators has real spectrum. As a consequence, we obtain the CF of asymptotically damped fields [4].

This result can also be generalized to stochastic fields:

$$
Z(x)=\exp \left(i \sum_{k=1}^{p} x_{k} A_{k}\right) Z_{0}
$$

where $x=\left(x_{1}, x_{2}, \ldots, x_{p}\right)$ and $A_{k}(k=1,2, \ldots, p)$ are commuting bounded linear operators defined in $H_{Z}$.

\section{References}

[1] L. Abbaoui, Application of the spectral theory of systems of nonself-adjoint linear operators to the study of nonhomogeneous random fields, Ph.D. thesis, Université d'Etat Maxime Gorki de Kharkov, 1984.

[2] M. S. Brodski1̆, Triangular and Jordan Representations of Linear Operators, American Mathematical Society, Rhode Island, 1971.

[3] M. S. Brodskiı̌ and M. S. Livšic, Spectral analysis of non-selfadjoint operators and intermediate systems, Amer. Math. Soc. Transl. (2) 13 (1960), 265-346.

[4] L. Debbi, Sur la Théorie Corrélative des Processus et des Champs Aléatoires Considérés comme des Courbes et des Surfaces dans un Espace de Hilbert, Mémoire de Magister, Université Ferhat Abbas, Sétif, 1994.

[5] I. C. Gohberg and M. G. Krein, Introduction to the Theory of Linear Nonselfadjoint Operators, Translations of Mathematical Monographs, vol. 18, American Mathematical Society, Rhode Island, 1969, translated from Russian by A. Feinstein.

[6] K. Ito, Veroyatnostnye Protsessy. I [Stochastic Processes. I], edited by E. B. Dynkin, Izdat. Inostr. Lit., Moscow, 1960, translated from Japanese by A. D. Vencel' and S. A. Verba.

[7] K. Kirchev and V. Zolotarev, Nonstationary curves in Hilbert spaces and their correlation functions. I, Integral Equations Operator Theory 19 (1994), no. 3, 270-289.

[8] M. S. Livšic and A. A. Yantsevich, Operator Colligations in Hilbert Spaces, John Wiley \& Sons, New York, 1979. 
[9] V. A. Zolotarev and A. A. Yantsevich, Nonstationary curves in Hilbert spaces and nonlinear operator equations, Operator Theory, Subharmonic Functions, Naukova Dumka, Kiev, 1991, pp. 54-60.

Lyazid Abbaoui: Department of Mathematics, University Ferhat Abbas, Elmaâbouda, Sétif 19000, Algeria

E-mail address: labbaoui@wissal.dz

Latifa Debbi: Department of Mathematics, University of M'sila, B.P 166, Echbilia, M'sila 28000, Algeria

E-mail address: 1debbi@yahoo.fr 


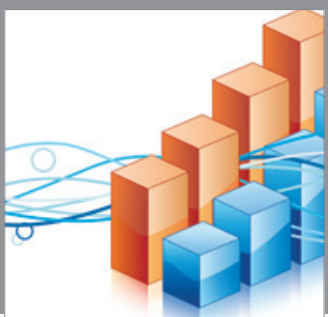

Advances in

Operations Research

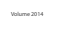

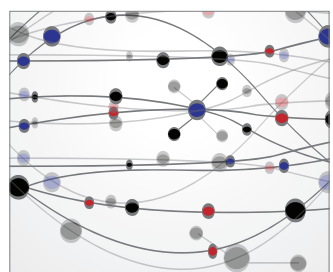

\section{The Scientific} World Journal
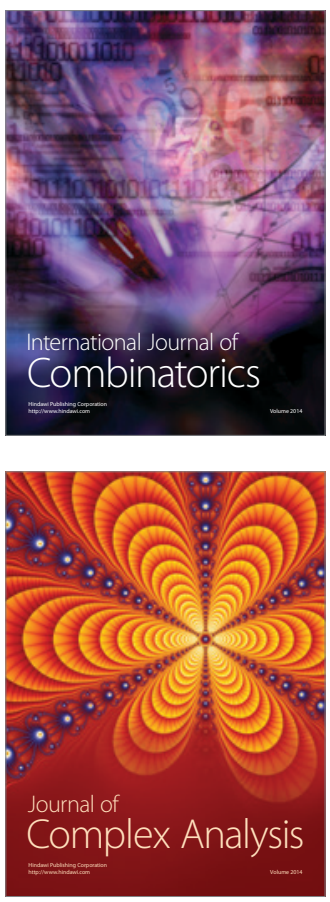

International Journal of

Mathematics and

Mathematical

Sciences
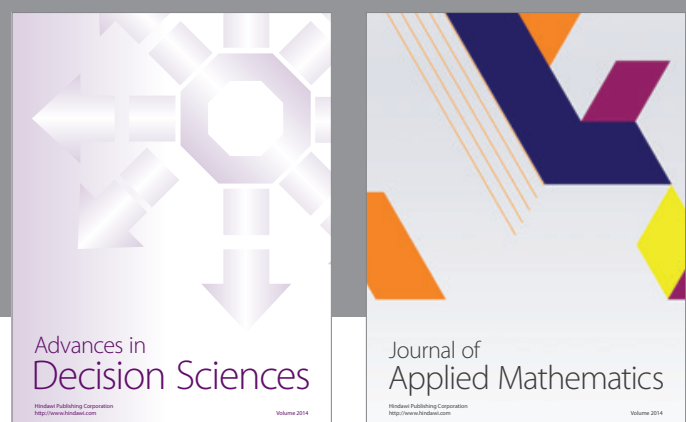

Journal of

Applied Mathematics
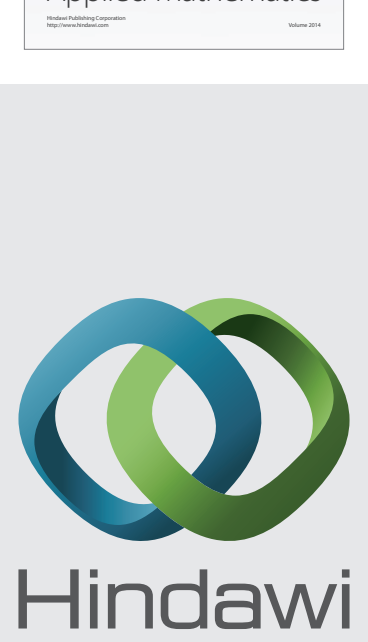

Submit your manuscripts at http://www.hindawi.com
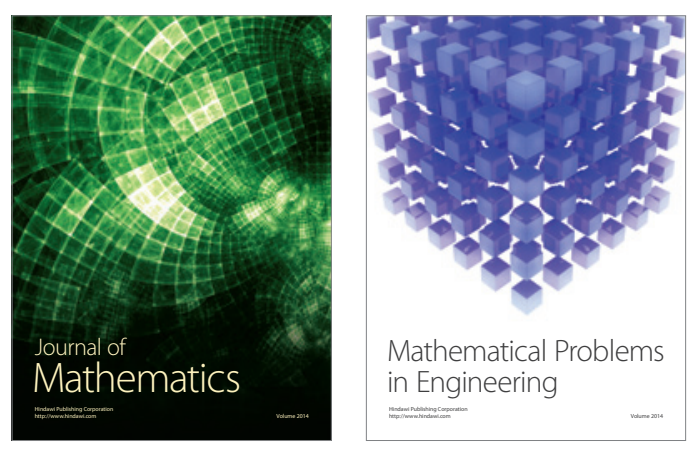

Mathematical Problems in Engineering
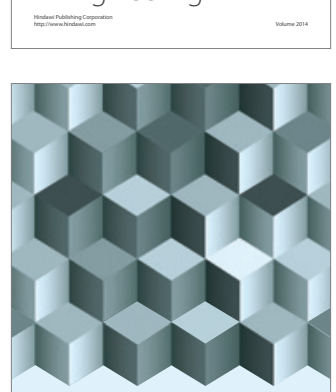

Journal of

Function Spaces
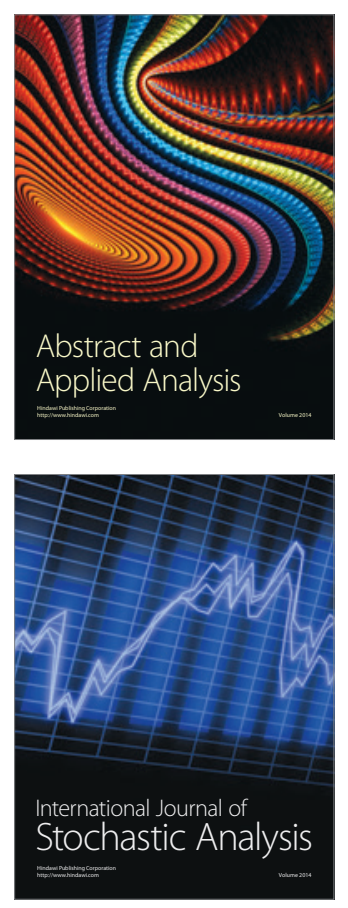

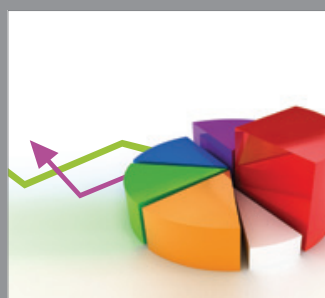

ournal of

Probability and Statistics

Promensencen
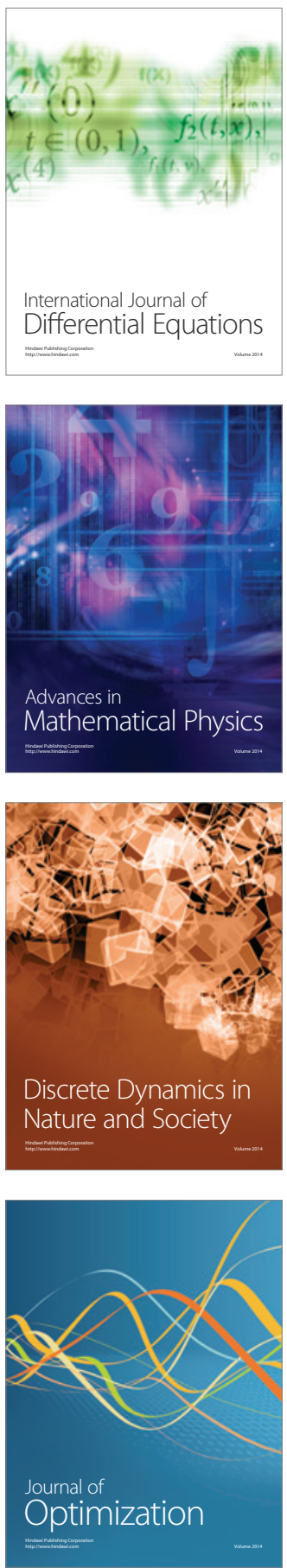